\title{
Effect of relative motion between bubbles and surrounding liquid on the Reynolds stress as a mechanism controlling the radial gas holdup distribution
}

\author{
K. Ueyama \\ Division of Chemical Engineering, \\ Graduate School of Engineering Science, Osaka University, Japan
}

\begin{abstract}
Relations are derived between time-averaged quantities of bubble turbulence, liquid velocity, static pressure and gravitational force, based on a careful treatment of the Navier-Stokes equations with certain approximations. The liquid phase is modeled as a combination of a bulk phase and a cloud phase. Timeintegration of the substantial derivative term in the Navier-Stokes equation for the cloud phase yields the difference between the total convective transport of liquid momentum from the cloud phase to the bulk phase and that from the bulk phase to the cloud phase, throughout the time of integration. The difference can be interpreted as bubble turbulence entering into the bulk phase, which should be convectively transported in the bulk phase. Finally, relations between timeaverages of the bubble turbulence, liquid velocity, static pressure and gravitational force are obtained through time-integration of the Navier-Stokes equation for the cloud phase. The resulting equation can be used to deduce, analytically or numerically, the macroscopic properties of gas-liquid multiphase flow in combination with a two-fluid model equation, for instance. These equations are applied to recirculating turbulent flow in bubble columns operating at high gas feed rates, and together with a simple model for the bubble turbulence we derive the well-known parabolic distribution of gas holdup.
\end{abstract}

Keywords: gas-liquid multiphase flow, macroscopic property, time-average, Navier-Stokes equation, cloud phase, gas holdup distribution, two-fluid model, recirculating turbulent flow. 


\section{Introduction}

Gas-liquid multiphase flow is widely used in the chemical, pharmaceutical and petrochemical industries to realize effective mass transfer, heat transfer and chemical reactions between gas and liquid phases. Gas-liquid multiphase flow in industrial apparatus usually involves a high gas volume fraction, to maintain high contact surface area between the two phases. Numerous studies have been made to determine and control the macroscopic properties of gas-liquid multiphase flow. Previous studies can be classified into three categories: numerical studies based on a two fluids model equation; approaches that build up from an understanding of the interaction between individual bubbles and the surrounding flow field; and studies of macroscopic properties of gas-liquid multiphase flow.

Numerical studies have become common as a result of the development of computer hardware and software. These works are based on a two-fluid model equation in which the interfacial force term comprises forces acting on a single sphere $[7,16]$. However, a volume-mean, for instance, of the interfacial force acting on bubble surfaces in a reference volume should reflect contributions of the forces acting on a bubble and the distribution of gas holdup. This argument is valid for any two-fluid model equation regardless of the details of the averaging. The interfacial force term in the two-fluid model equation therefore should represent the combined effect of forces acting on a bubble and the spatial distribution of gas holdup.

The approach that proceeds from an understanding of the interaction between a single bubble and the surrounding flow field has already been helpful in studying dynamics of a single bubble in various flow fields $[4,5,8]$. This approach needs adapting to deal with gas-liquid multiphase flow with relatively high gas hold up and the effects of surrounding bubbles.

Studies based on macroscopic properties of gas-liquid multiphase flow proceed from known macroscopic properties to provide an understanding of basic phenomena such as interaction between a single bubble and the surrounding flow field. Sato et al. [6] introduced the notion of bubble turbulence, and showed that the measured radial distribution of the mean liquid velocity accurately coincides with model prediction based on the measured radial distribution of gas holdup together with the eddy diffusivity for fully developed turbulent flow and the calculated Reynolds stress due to bubble turbulence. The success of this model implies that bubble turbulence is important in determining macroscopic properties of gas-liquid multiphase flow. Zhang and Ahmadi [16] showed that a modification of $\kappa-\varepsilon$ model taking bubble turbulence into account could accurately reproduce the time-averaged velocity profile in bubbly flow based on a two-fluid model equation.

In the present work, a phenomenological definition of bubble turbulence is given by time-averaging the Navier-Stokes equations as proposed by Ueyama and Miyauchi [9], for a cloud phase surrounding the bubbles. A general relation between bubble turbulence and turbulent flow quantities is derived which can be used to deduce macroscopic properties of gas-liquid multiphase flow. The 
assumptions used to obtain this relation are analyzed and shown to be acceptable. Finally, the well-known parabolic distribution of gas holdup for recirculating turbulent flow in bubble columns operated at a high gas feed rate $[2,3,10-12,15]$ is obtained as an analytical solution of our relation.

\section{Definition and notation for time-averaging}

We can time-integrate the Navier-Stokes equations, at a fixed point in space, for the bubble phase, liquid phase or for any fixed quantity of fluid we define mathematically by its bounding surface. The time-averaged Navier-Stokes equations for the bubble or liquid phase give relations between time-averaged physical quantities for those phases.

Turbulence induced by bubble motion relative to the surrounding liquid, henceforth referred to as bubble turbulence, plays an important role in a mechanism controlling the gas holdup distribution [6,16]. Let us consider the liquid phase as comprising a cloud phase and a bulk phase in discussing the effect of bubble turbulence on the mechanism of gas-liquid multiphase flow. We suppose that each bubble is surrounded by a cloud, in which bubble turbulence is generated by relative motion between the bubble and liquid. At this point we are not concerned whether the cloud includes multiple bubbles and the physical definition of the cloud is not necessary for the time-averaging procedure.

Bellow, the notations involved in the time-averaging procedure are set out, and the definition of the cloud phase is stated. Time-integration is performed at a fixed point in space over a sufficiently long interval $\Lambda$ to obtain reliable timeaveraged values. The gradient of a physical quantity $q$ can be time-averaged as in eqn (1).

$$
\begin{aligned}
& \frac{1}{\Lambda} \int_{t}^{t+\Lambda} \nabla q d t \equiv \overline{\nabla q}^{\Lambda}=\frac{\Lambda_{c}}{\Lambda} \overline{\nabla q}^{c}=\frac{1}{\Lambda} \int_{\Lambda_{c}} \nabla q d t \\
= & \nabla\left(\frac{\Lambda_{c}}{\Lambda} \bar{q}^{c}\right)+\frac{1}{\Lambda}\left[\sum_{i=1}^{N}\left\{\left.(q \xi)\right|_{t=T_{i}^{l}}-\left.(q \xi)\right|_{t=T_{i}^{a}}\right\}+\sum_{j=1}^{M}\left\{\left.(q \xi)\right|_{t=T_{j}^{\prime a}}-\left.(q \xi)\right|_{t=T_{j}^{\prime \prime}}\right\}\right]
\end{aligned}
$$

Here, the suffix $c$ refers to the cloud phase and the superscript ${ }^{-\Lambda}$ denotes the time-averaged value over a time interval $\Lambda$. A notation $\Lambda_{c}$ denotes the sum of individual time lengths during which the cloud phase is continuously observed. A superscript ${ }^{-c}$ refers to the value averaged over a time interval $\Lambda_{c}$, and $\int_{\Lambda_{c}} d t$ refers to integration over the time during which the cloud phase is continuously observed. We denote by $T_{i}^{a}$ and $T_{i}^{l}$ the arriving and leaving time of the $i$-th bubble. Notations $T_{j}^{\prime a}$ and $T^{\prime l}{ }_{j}$ respectively denote the arriving and leaving time of the $j$-th cloud. The values of $T_{i}^{a}, T_{i}^{l}, T_{j}^{\prime a}$ and $T^{\prime l}{ }_{j}$ depend on position. The symbols $M$ and $N$ denote the total numbers of clouds and bubbles, 
respectively. The vector $\xi$ is the gradient vector of the surface $t=T_{i}^{a}, t=T_{i}^{l}$, $t=T_{j}^{\prime a}$ or $t=T_{j}^{\prime l}$.

$$
\xi=\nabla T
$$

If $\mathbf{u}_{s}$ is a moving velocity vector of bubble surface, it follows that:

$$
\mathbf{u}_{s} \cdot \xi=1
$$

If there is no mass transfer across the surface, the following eqn (4) is obtained, because $\xi$ is normal to the surface.

$$
\left(\mathbf{u}-\mathbf{u}_{s}\right) \cdot \boldsymbol{\xi}=0
$$

In the absence of mass transfer across the surface, it then follows that:

$$
\mathbf{u} \cdot \xi=1
$$

\section{Time-integrations of terms in the Navier-Stokes equations}

\subsection{Substantial derivative term}

Upon applying eqns (3), (4) and (5), a substantial derivative term can be timeaveraged, as follows:

$$
\begin{aligned}
& \frac{1}{\Lambda} \int_{\Lambda_{c}}\left\{\frac{\partial}{\partial t}(\rho \mathbf{u})+\nabla \cdot(\rho \mathbf{u u})\right\} d t \\
& =\nabla \cdot\left(\frac{\Lambda_{\mathbf{c}}}{\Lambda} \overline{\rho \mathbf{u u}}^{c}\right)-\frac{1}{\Lambda} \sum_{i=1}^{N}\left\{\left.(\rho \mathbf{u})\right|_{T_{i}^{l}}-\left.(\rho \mathbf{u})\right|_{T_{i}^{a}}\right\}+\frac{1}{\Lambda} \sum_{i=1}^{N}\left\{\left.(\rho \mathbf{u u})\right|_{T_{i}^{l}} \cdot \boldsymbol{\xi}_{T_{i}^{l}}-\left.(\rho \mathbf{u u})\right|_{T_{i}^{a}} \cdot \boldsymbol{\xi}_{T_{i}^{a}}\right\} \\
& -\frac{1}{\Lambda} \sum_{j=1}^{M}\left\{\left.(\rho \mathbf{u})\right|_{T_{j}^{\prime a}}-\left.(\rho \mathbf{u})\right|_{T_{j}^{\prime l}}\right\}+\frac{1}{\Lambda} \sum_{j=1}^{M}\left\{\left.(\rho \mathbf{u u})\right|_{T_{j}^{\prime a}} \cdot \boldsymbol{\xi}_{T^{\prime a}}-\left.(\rho \mathbf{u u})\right|_{T_{j}^{\prime{ }_{j}}} \cdot \boldsymbol{\xi}_{T^{\prime \prime}{ }_{j}}\right\} \\
& =\nabla \cdot\left(\frac{\Lambda_{\mathrm{c}}}{\Lambda} \overline{\rho \mathbf{u u}}^{c}\right)-\frac{1}{\Lambda} \sum_{j=1}^{M}\left[\left\{\rho \mathbf{u}\left(\mathbf{u}-\mathbf{u}_{s}\right)\right\}_{T^{\prime \prime}{ }_{j}} \cdot \boldsymbol{\xi}_{T^{\prime^{l}}{ }_{j}}-\left\{\rho \mathbf{u}\left(\mathbf{u}-\mathbf{u}_{s}\right)\right\}_{T^{\prime}{ }_{j}} \cdot \boldsymbol{\xi}_{T^{\prime a}}\right]
\end{aligned}
$$

Here $\rho$ and $\mathbf{u}$ respectively denote the density and velocity vector of the liquid. A vector $\xi_{T^{\prime}{ }_{j}^{a}}$ can be rewritten using the normal unit vector $\mathbf{n}$ at the surface, as:

$$
\xi_{T_{j}^{\prime a}}=\frac{\mathbf{n}}{\left(\mathbf{u}_{s} \cdot \mathbf{n}\right)_{T^{\prime}{ }_{j}}}
$$

Let us choose the direction of $\mathbf{n}$ toward the movement of the cloud surface, in order to interpret the second term on the right hand side of the final term in eqn (6). The term $-\left\{\rho \mathbf{u}\left(\mathbf{u}-\mathbf{u}_{s}\right)\right\}_{T^{\prime a}{ }_{j}} \cdot \xi_{T^{\prime}{ }_{j}}$ can be rewritten as:

$$
-\left\{\rho \mathbf{u}\left(\mathbf{u}-\mathbf{u}_{S}\right)\right\}_{T^{\prime a}} \cdot \xi_{T_{j}^{\prime a}}=\frac{-\left\{\rho \mathbf{u}\left(\mathbf{u}-\mathbf{u}_{s}\right)\right\}_{T_{j}^{\prime}{ }_{j}} \cdot \mathbf{n}}{\left(\mathbf{u}_{s} \cdot \mathbf{n}\right)_{T_{j}^{\prime a}}}
$$

Since liquid is flowing into the cloud phase from the bulk phase, and the cloud surface is moving towards the bulk phase at $t=T_{j}^{\prime a}$, the numerator on the 
right hand side of eqn (8) is the flux of momentum transported convectively from the bulk phase to the cloud phase. The denominator is the volume swept by the surface per unit time. The right hand side of eqn (8) can be understood as the total impulses, divided by the total time, per unit volume added at the instance when the cloud arrives at the time-averaging point, that is due to convective transport of momentum from the bulk phase to the cloud phase.

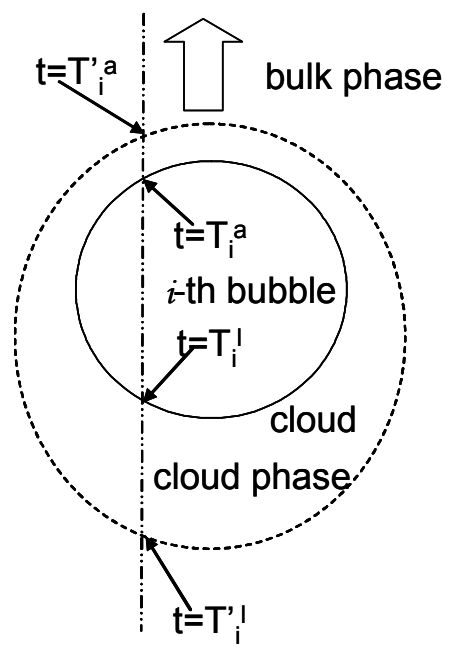

Figure 1: Schematic diagram of the bubble, cloud and bulk phases.

We thus conclude that the second term on the right hand side of the final term in eqn (6) is the difference between liquid momentum transported convectively from the cloud phase to the bulk phase and that from the bulk phase to the cloud phase; that is, the convective transport term of the bubble turbulence momentum, $\rho \mathbf{u}_{b}^{\prime}$, into the bulk phase; this represents an input of bubble turbulence momentum to the bulk phase.

The input of bubble turbulence momentum is immediately transported by liquid flow in the bulk phase, and it should therefore be balanced with the timeaveraged convective transport of bubble turbulence, $-\nabla \cdot \Lambda_{b} \rho{\overline{\mathbf{u}_{b}^{\prime}}}^{b}$, for the bulk phase. Here, $\Lambda_{b}$ is a sum of individual time durations during which the bulk phase is continuously observed. The superscript ${ }^{-b}$ signifies a time-averaged value over the time interval $\Lambda_{b}$. The balance between input and convective transport terms for bubble turbulence is expressed as:

$$
0=-\sum_{j=1}^{M}\left[\left\{\rho \mathbf{u}\left(\mathbf{u}-\mathbf{u}_{S}\right)\right\}_{T^{\prime \prime}{ }_{j}} \cdot \boldsymbol{\xi}_{T^{\prime \prime}{ }_{j}}-\left\{\rho \mathbf{u}\left(\mathbf{u}-\mathbf{u}_{s}\right)\right\}_{T^{\prime a}{ }_{j}} \cdot \boldsymbol{\xi}_{T_{j_{j}}}\right]-\nabla \cdot \Lambda_{b} \rho{\overline{\mathbf{u}_{b}{ }_{b} \mathbf{u}}}^{b}
$$

From eqns (6) and (9), time-integration of the substantial derivative term leads to eqn (10). 


$$
\begin{aligned}
& \frac{1}{\Lambda} \int_{\Lambda_{c}}\left\{\frac{\partial}{\partial t}(\rho \mathbf{u})+\nabla \cdot(\rho \mathbf{u u})\right\} d t \\
= & \nabla \cdot\left(\frac{\Lambda_{c}}{\Lambda} \rho \overline{\mathbf{u u}}^{c}\right)+\nabla \cdot\left(\frac{\Lambda_{b}}{\Lambda} \rho{\overline{\mathbf{u}_{b}^{\prime} \mathbf{u}^{b}}}^{b}\right) \\
= & \nabla \cdot\left(\frac{\Lambda_{c}}{\Lambda} \rho \rho_{\mathbf{u}}^{-l-l}\right)+\nabla \cdot\left(\frac{\Lambda_{c}}{\Lambda} \rho{\overline{\mathbf{u}^{\prime}{ }_{L} \mathbf{u}^{\prime}}}^{c}\right)+\nabla \cdot\left(\frac{\Lambda_{c}}{\Lambda} \rho{\overline{\mathbf{u}^{\prime}{ }_{b} \mathbf{u}_{b}^{\prime}}}^{c}\right)+\nabla \cdot\left(\frac{\Lambda_{b}}{\Lambda} \rho_{{\overline{\mathbf{u}^{\prime}}}_{b} \mathbf{u}_{b}^{\prime}}^{b}\right)
\end{aligned}
$$

Here, $\mathbf{u}_{L}^{\prime}$ is turbulence other than the bubble turbulence $\mathbf{u}_{b}^{\prime}$, and is assumed to be independent of the bubble turbulence. The superscript ${ }^{-l}$ refers as usual to a time-averaged value over the time interval $\Lambda_{l}=\Lambda_{b}+\Lambda_{c}$, that is the entire duration during which the liquid phase is observed.

$$
\mathbf{u}=\overline{\mathbf{u}}^{l}+\mathbf{u}^{\prime}=\overline{\mathbf{u}}^{l}+\mathbf{u}_{b}{ }_{b}+\mathbf{u}^{\prime}{ }_{L},
$$

In eqn. (11), $\mathbf{u}^{\prime}$ is a fluctuating component of the liquid velocity.

Eqn (12) holds in the cloud phase, because the bubble turbulence is newly generated there:

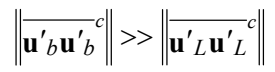

By applying the condition (12) in eqn (10), we find that:.

$$
\frac{1}{\Lambda} \int_{\Lambda_{c}}\left\{\frac{\partial}{\partial t}(\rho \mathbf{u})+\nabla \cdot(\rho \mathbf{u u})\right\} d t \approx \nabla \cdot\left(\frac{\Lambda_{c}}{\Lambda} \rho \overline{\mathbf{u}}^{-l-l}\right)+\nabla \cdot\left(\frac{\Lambda_{l}}{\Lambda} \rho_{\overline{\mathbf{u}_{b}^{\prime} \mathbf{u}^{\prime} b}}^{l}\right)
$$

The second term on the right hand side of eqn. (13) is obtained from the general property of time-averaging. The right hand side is given by the physical components time-averaged over the entire duration for which the liquid phase is observed. This will enable us to construct a simple physical model for the bubble turbulence.

\subsection{Static pressure term}

We have:

$$
-\frac{1}{\Lambda} \int_{\Lambda_{c}}(\nabla P) d t=-\nabla\left(\frac{\Lambda_{c}}{\Lambda} \bar{P}^{c}\right)+\frac{1}{\Lambda} \sum_{i=1}^{N}\left(\left.P\right|_{T_{i}^{a}} \xi_{T_{i}^{a}}-\left.P\right|_{T_{i}^{\prime}} \boldsymbol{\xi}_{T_{i}^{l}}\right)+\frac{1}{\Lambda} \sum_{j=1}^{M}\left(\left.P\right|_{T^{\prime} j_{j}} \boldsymbol{\xi}_{T_{j}^{\prime_{j}^{\prime}}}-\left.P\right|_{T_{T_{j}^{\prime}} \boldsymbol{\xi}_{T^{\prime a}}}\right)
$$

The second and third terms on the right hand side are surface terms representing the effect of static pressure at the bubble surface and cloud surface, respectively. In the conventional two-fluid model equation, the surface term for the static pressure acting on unit volume of a particular phase is given as the product of the mean static pressure and the gradient of a local fraction of the phase;

$$
\frac{1}{\Lambda}\left\{\sum_{i=1}^{N}\left(\left.P\right|_{T_{i}^{a}} \xi_{T_{i}^{a}}-\left.P\right|_{T_{i}^{l}} \xi_{T_{i}^{l}}\right)+\sum_{j=1}^{M}\left(\left.P\right|_{T^{\prime \prime}{ }_{j}} \xi_{T^{\prime \prime}{ }_{j}}-\left.P\right|_{T^{\prime a}} \xi_{T^{\prime}{ }_{j}^{a}}\right)\right\}=\bar{P}^{c} \nabla\left(\frac{\Lambda_{c}}{\Lambda}\right)
$$

From eqns.(14) and (15), it follows that:

$$
-\frac{1}{\Lambda} \int_{\Lambda_{c}}(\nabla P) d t=-\nabla\left(\frac{\Lambda_{c}}{\Lambda} \bar{P}^{c}\right)+\bar{P}^{c} \nabla\left(\frac{\Lambda_{c}}{\Lambda}\right)=-\frac{\Lambda_{c}}{\Lambda}\left(\nabla \bar{P}^{c}\right)
$$




\subsection{Stress tensor term}

We have:

$$
\begin{aligned}
& -\frac{1}{\Lambda} \int_{\Lambda_{c}}(\nabla \cdot \boldsymbol{\tau}) d t \\
= & -\nabla \cdot\left(\frac{\Lambda_{c}}{\Lambda}-\bar{\tau}\right)+\frac{1}{\Lambda} \sum_{i=1}^{N}\left(\left.\boldsymbol{\tau}\right|_{T_{i}^{a}} \cdot \boldsymbol{\xi}_{T_{i}^{a}}-\left.\boldsymbol{\tau}\right|_{T_{i}^{l}} \cdot \boldsymbol{\xi}_{T_{i}^{l}}\right)+\frac{1}{\Lambda} \sum_{j=1}^{M}\left(\left.\boldsymbol{\tau}\right|_{T^{\prime \prime}} \cdot \boldsymbol{\xi}_{T^{\prime \prime} j_{j}}-\left.\boldsymbol{\tau}\right|_{T^{\prime}{ }_{j}} \cdot \boldsymbol{\xi}_{T^{\prime a}}\right)
\end{aligned}
$$

The second term on the right hand side is a surface term due to the viscous force at the bubble surface. This can be calculated by time-averaging the NavierStokes equations for the gas phase in gas-liquid multiphase flow, neglecting the density and viscosity terms $[9,13,14]$ :

$$
\frac{1}{\Lambda} \sum_{i=1}^{N}\left(\boldsymbol{\tau}_{T_{i}^{a}} \cdot \boldsymbol{\xi}_{T_{i}^{a}}-\boldsymbol{\tau}_{T_{i}^{\prime}} \cdot \boldsymbol{\xi}_{T_{i}^{T_{i}}}\right) \approx-\frac{\Lambda-\Lambda_{l}}{\Lambda}(\nabla \bar{P})
$$

The third term on the right hand side of eqn (17) is a surface term due to the shear stress at the cloud surface. It should cancel with the effect of shear stress at the bubble surface when the cloud thickness is negligible. The third term vanishes when the cloud phase entirely occupies the liquid phase. Hence, the third term can be approximated as:

$$
\frac{1}{\Lambda} \sum_{j=1}^{M}\left(\left.\tau\right|_{T^{\prime \prime}} \cdot \xi_{T_{j}^{\prime l}}-\left.\tau\right|_{T_{j}^{\prime a}} \cdot \xi_{T^{\prime a}}\right) \approx \frac{\Lambda_{l}-\Lambda_{c}}{\Lambda} \frac{\Lambda-\Lambda_{l}}{\Lambda_{l}}(\nabla \bar{P})
$$

The right hand side goes to $\frac{\Lambda-\Lambda_{l}}{\Lambda}\left(\nabla \bar{P}^{l}\right)$ when $\Lambda_{c}$ vanishes, and is zero when $\Lambda_{c}=\Lambda_{l}$. By substituting eqns. (18) and (19) into eqn (17), we have:

$$
-\frac{1}{\Lambda} \int_{\Lambda_{c}}(\nabla \cdot \tau) d t \approx-\nabla \cdot\left(\frac{\Lambda_{c}}{\Lambda} \bar{\tau}^{c}\right)-\frac{\Lambda_{c}\left(\Lambda-\Lambda_{l}\right)}{\Lambda \Lambda_{l}}\left(\nabla \bar{P}^{l}\right)
$$

\subsection{Gravitation term}

We have:

$$
\frac{1}{\Lambda} \int_{\Lambda_{c}}(\rho \mathbf{g}) d t=\frac{\Lambda_{c}}{\Lambda} \rho \mathbf{g}
$$

\section{Time-integrations of the Navier-Stokes equations for the cloud phase}

We now have, from eqns.(13), (16), (20) and (21):

$$
\nabla \cdot\left(\frac{\Lambda_{c}}{\Lambda} \rho_{\mathbf{u} \mathbf{u}}^{-l-l}\right)+\nabla \cdot\left(\frac{\Lambda_{l}}{\Lambda} \rho \overline{\mathbf{u}_{b}^{\prime} \mathbf{u}_{b}^{\prime}}\right)=-\frac{\Lambda_{c}}{\Lambda_{l}}\left(\nabla \bar{P}^{l}\right)-\nabla \cdot\left(\frac{\Lambda_{c}}{\Lambda} \overline{\boldsymbol{\tau}}^{c}\right)+\frac{\Lambda_{c}}{\Lambda} \rho \mathbf{g}
$$

The absolute value of the second term on the right hand side is usually very small compared to that of the second term on the left hand side, the first term on the right hand side, or the third term on the right hand side, in which case: 


$$
\nabla \cdot\left(\frac{\Lambda_{c}}{\Lambda} \rho_{\mathbf{u}}^{-l-l} \mathbf{u}\right)+\nabla \cdot\left(\frac{\Lambda_{l}}{\Lambda} \rho \overline{\mathbf{u}_{b}^{\prime} \mathbf{u}^{\prime} b}\right) \approx \frac{\Lambda_{c}}{\Lambda_{l}}\left(-\nabla \bar{P}^{l}+\frac{\Lambda_{l}}{\Lambda} \rho \mathbf{g}\right)
$$

Ueyama has derived the following equation from the Navier-Stokes equations time-averaged for the liquid phase in gas-liquid multiphase flow $[9,13,14]$ :

$$
\nabla \bar{P}^{l}=-\nabla \cdot\left(\frac{\Lambda_{l}}{\Lambda} \overline{\rho \mathbf{u u}}^{l}\right)-\nabla \cdot\left(\frac{\Lambda_{l}}{\Lambda} \overline{\boldsymbol{\tau}}^{l}\right)+\frac{\Lambda_{l}}{\Lambda} \rho \mathbf{g}
$$

By substituting eqn (24) into eqn (23), and neglecting the second terms on the right hand sides of eqn(24) in comparison with the Reynolds stress term in the first term on the right hand side of eqn (24), we have:

$$
\nabla \cdot\left(\frac{\Lambda_{c}}{\Lambda} \rho_{\mathbf{u}}^{-l-l} \mathbf{u}\right)+\nabla \cdot\left(\frac{\Lambda_{l}}{\Lambda} \rho{\overline{\mathbf{u}^{\prime}{ }_{b} \mathbf{u}^{\prime} b}}^{l}\right) \approx \frac{\Lambda_{c}}{\Lambda_{l}} \nabla \cdot\left(\frac{\Lambda_{l}}{\Lambda} \rho \overline{\mathbf{u u}}^{l}\right)
$$

The gravitation term cancels in deriving eqn (25). The physical quantities in eqns (23) and (25) are all time-averaged values over the time interval $\Lambda_{l}$, which is the total duration during which the liquid phase is observed. Eqn (23) gives a relation between bubble turbulence, static pressure and gravitational force, and eqn (25) is a relation between the tensors $-\Lambda^{\Lambda_{l}}-\Lambda^{\Lambda_{l}}, \overline{\mathbf{u}_{b}^{\prime} \mathbf{u}_{b}^{\prime}} \Lambda_{l}$ and $\overline{\mathbf{u u}} \Lambda^{\Lambda_{l}}$. In combination with the two-fluid model equation, eqns (23) and (25) allow us to deduce the macroscopic properties of gas-liquid multiphase flow.

\section{Gas holdup distribution for recirculating turbulent flow}

In this section, eqn (23) is applied to recirculating turbulent flow in large scale bubble columns operating at high gas feed rates. The radial and angular component of the time-averaged velocity are both zero, the axial and angular gradient of time-averaged quantities are zero, and no stationary swirl flow is observed in such a flow field [2,3,10-12,15]. The radial distribution of gas holdup for the recirculating turbulent flow can be expressed as:

$$
\varepsilon=\varepsilon_{0}\left(1-\phi^{n}\right)
$$

Table 1: $\quad$ Values of $n$ for the gas holdup distribution.

\begin{tabular}{|l|c|c|c|c|}
\hline$D_{\mathrm{T}}[\mathrm{m}]$ & $U_{\mathrm{G}}[\mathrm{m} / \mathrm{s}]$ & gas distributor & $n$ & reference \\
\hline 0.1 & 0.05 & single nozzle & 1.6 & {$[15]$} \\
\hline 0.138 & $0.02-0.17$ & perforated plate & $1.6-1.8$ & {$[2]$} \\
\hline 0.12 & $0.02-0.15$ & perforated plate & $1.7-2.5$ & {$[3]$} \\
\hline 0.3 & 0.46 & single nozzle & 1.5 & {$[10]$} \\
\hline 0.6 & $0.35-0.93$ & single nozzle & $1.8-2.3$ & {$[10]$} \\
\hline
\end{tabular}

Here, $\varepsilon$ is the gas holdup defined as the ratio $\Lambda_{1} / \Lambda$, and $\varepsilon_{0}$ is the gas holdup at the center of the column. We define a dimensionless radial coordinate $\phi=r / R$, 
and $n$ is a fitting parameter. Ueyama $[10,11]$ obtained numerical values of $n$ which fit the measured distribution of gas holdup, as shown in Table 1.

The values of $n$ in Table 1 are scattered around $n=2$.

Eqn. (27) was obtained from the Navier-Stokes equations, time-averaged for gas-liquid multiphase flow [13].

$$
(1-\varepsilon) \rho_{c} \overline{u_{r}^{\prime}{ }_{r}^{\prime}{ }_{z}^{\prime}} \lambda_{c}=-\frac{R}{\phi} \int_{0}^{\phi} \phi\left\{\left(\varepsilon_{m}-\varepsilon\right) \rho_{c} g-\frac{2}{R} \tau_{w}\right\} d \phi
$$

Here, $\varepsilon_{m}$ is a cross sectional mean of the gas holdup, $\varepsilon_{W}$ is the gas holdup at the column wall, and $\tau_{W}$ denotes the shear stress. Ueyama and Saitoh [14] recently showed that the radial distributions of the Reynolds stress measured by Degaleesan [1] agree very well with eqn. (27) for the parabolic distribution of gas holdup, as shown in Figure 2.

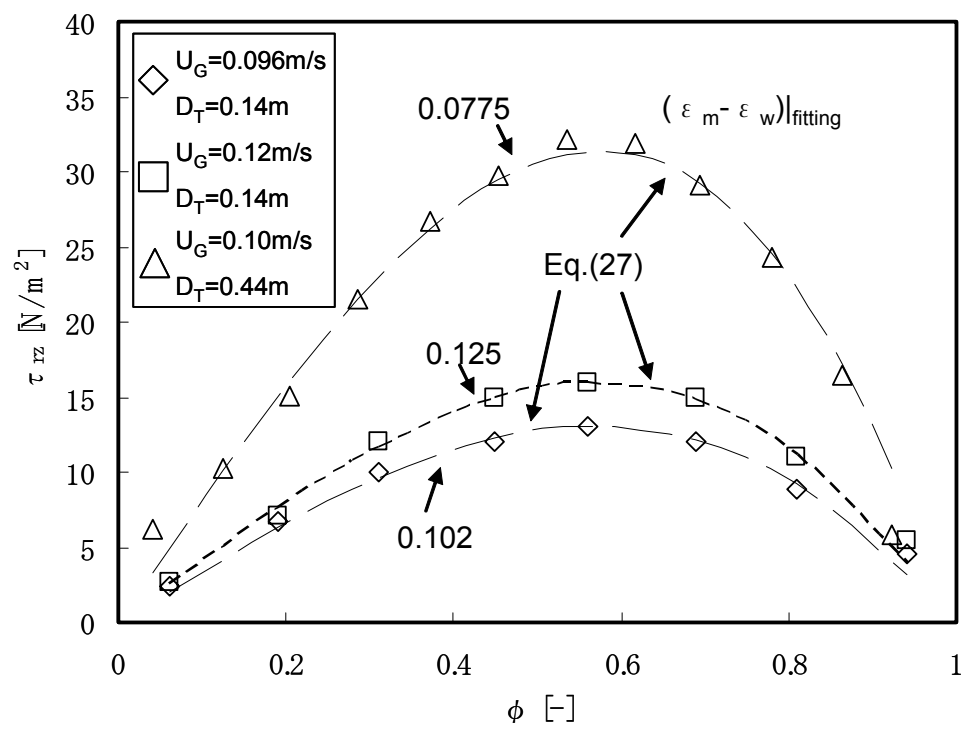

Figure 2: $\quad$ Prediction based on a parabolic gas holdup distribution and Degaleesan's data [1] for the radial distribution of Reynolds stress.

It can be concluded that the radial gas holdup distribution is parabolic for the recirculating turbulent flow in bubble columns. Below, we shall derive the parabolic distribution from eqn. (23).

Eqn. (28) is obtained from Navier-Stokes equation time-averaged for gasliquid multiphase flow $[9,13,14]$.

$$
\frac{\partial \bar{P}^{l}}{\partial z}=-\frac{2}{R} \tau_{W}-\left(1-\varepsilon_{m}\right) \rho g
$$

The axial component of eqn (23) becomes, upon exploiting eqn. (28): 


$$
\frac{1}{r} \frac{\partial}{\partial r}\left\{(1-\varepsilon) \rho r_{\overline{u_{b r}^{\prime} u_{b z}^{\prime}} l}\right\} \approx \alpha\left\{\frac{2}{R} \tau_{W}+\left(\varepsilon-\varepsilon_{m}\right) \rho g\right\}
$$

Here, $\alpha=\Lambda_{c} / \Lambda_{l}$. The term $(1-\varepsilon) \overline{u_{b r}^{\prime} u_{b z}^{\prime}}$ on the left hand side of eqn (29) is defined as follows:

$$
(1-\varepsilon){\overline{u_{b r}^{\prime} u_{b z}^{\prime}}}^{l}=\frac{1}{\Lambda} \int_{\Lambda_{l}} u_{b r}^{\prime} u_{b z}^{\prime} d t
$$

The value of this expression depends strongly on the motion of bubbles surrounding the spatial point at which time-averaging is performed. We now construct a simple model to deduce the value of the left hand side, using a Cartesian coordinate system for gas-liquid multiphase flow with gas holdup distributed in the $x$-direction and homogeneous in the $y$ - and $z$-direction. When bubbles are rising, in the $z$-direction, the value of $\int u_{b x}^{\prime} u_{b z}^{\prime} d t$ consists of a $\Lambda_{l}$

contribution due to bubbles which have passed through the $x^{+}$region, which is a region with $x$-value greater than that of the time-averaging point, and a contribution from bubbles in the $x^{-}$region. Represent the contribution of the $x^{+}$bubbles as $F\{\varepsilon(x+\lambda)\}$, where $\varepsilon(x+\lambda)$ is the gas holdup at $x+\lambda$. The contribution of the $x^{-}$bubbles can be expressed as $-F\{\varepsilon(x-\lambda)\}$, since the sign of the bubble turbulence velocity, $u_{b x}^{\prime}$, to be coupled with $u_{b z}^{\prime}$ is opposite to that of the contribution of the $x^{+}$bubbles for the same value of $u_{b z}^{\prime}$. Hence, the value of $\frac{1}{\Lambda} \int_{\Lambda_{l}} u_{b x}^{\prime} u_{b z}^{\prime} d t$ is given as:

$$
\begin{aligned}
\frac{1}{\Lambda} \int_{\Lambda_{l} u_{b r} u_{b z}^{\prime} d t} & =F\{\varepsilon(x+\lambda)\}-F\{\varepsilon(x+\lambda)\} \\
& \left.\approx 2 \lambda \frac{\partial}{\partial x}\right|_{x=x} F\{\varepsilon(x)\}=\left.\left.\frac{\partial \varepsilon}{\partial x}\right|_{x=x} f(\varepsilon)\right|_{\varepsilon=\varepsilon(x)}
\end{aligned}
$$

Here, $f(\xi) \equiv 2 \lambda \frac{d}{d \varepsilon} F(\varepsilon)$. Eqn (31) is different from the model proposed by Sato et al. [6], which took the value to be proportional to the gas holdup $\varepsilon$ : however, the present model may fit the phenomenon better.

By substituting eqn (31) into eqn (29), we have:

$$
\frac{d}{d \phi}\left\{\phi\left(\frac{d \varepsilon}{d \phi}\right) f(\varepsilon)\right\} \approx \alpha \phi\left\{2 \frac{\tau_{W}}{\rho}+\left(\varepsilon-\varepsilon_{m}\right) R g\right\}
$$

Upon taking a polynomial expression for $\varepsilon$ and $f(\varepsilon)$, the following solutions are obtained in the case where the parameter $\alpha$ is constant.

$$
\varepsilon=\varepsilon_{0}+\left(\varepsilon_{W}-\varepsilon_{0}\right) \phi^{2}
$$




$$
f(\varepsilon)=\alpha \frac{R g}{8\left(\varepsilon_{W}-\varepsilon_{0}\right)}\left\{4 \frac{\tau_{W}}{\rho R g}+\left(\varepsilon-\varepsilon_{W}\right)\right\}
$$

Eqn. (33) gives the parabolic radial distribution of gas holdup, which has been known empirically for forty years. The most dubious assumption involved in deriving the parabolic distribution is that the value of $\alpha=\Lambda_{c} / \Lambda_{l}$ is constant regardless of the gas holdup value. However, the assumption of constant $\alpha$ is acceptable because, even if the value of $\alpha$ depends on the gas holdup, there should be a minimum positive value of $\alpha$ which we can adopt in deriving eqn. (29).

\section{Conclusion}

The Navier-Stokes equations have been time-integrated for the cloud phase which surrounding each bubble.

A phenomenological definition of bubble turbulence is clearly introduced as the liquid momentum generated in the cloud phase.

It is also shown that the bubble turbulence generated in the cloud phase acts as an input of liquid momentum in the bulk phase, and is in balance with convective transport in the bulk phase.

Eqs. (23) and (25) above show the relation between the bubble turbulence and the time-averaged velocity field of gas-liquid multiphase flow.

By applying eqn. (23) to the recirculating turbulent flow in bubble columns, the well-known parabolic radial distribution of gas hold up is obtained as an analytical solution.

Eqns. (23) and (25) can be applied to any kind of gas-liquid multiphase flow, so as to deduce macroscopic properties of the multiphase flow.

\section{Symbols}

$D_{T}: \quad$ column diameter

g: gravitational acceleration

g : vector of gravitational acceleration vector

$M: \quad$ total number of clouds

$n: \quad$ fitting parameter in eqn. (27)

$N: \quad$ total number of bubbles

$P: \quad$ static pressure

$q: \quad$ physical quantity

$r: \quad$ radial coordinate

$R$ : $\quad$ column radius

$t: \quad$ time

$T_{i}^{a}: \quad$ arrival time of the $i$-th bubble 
$T_{i}^{l}: \quad$ time of leaving of the $i$-th bubble $[s]$

$T^{\prime a}{ }_{j}^{a}: \quad$ arrival time of $j$-th cloud $[s]$

$T^{\prime l}{ }_{j}:$ time of leaving of the $j$-th cloud $[s]$

$u:$ velocity $\left[\mathrm{m} \cdot \mathrm{s}^{-1}\right]$

$\mathbf{u}:$ velocity vector $\left[\mathrm{m} \cdot \mathrm{s}^{-1}\right]$

$\mathbf{u}^{\prime}$ : fluctuating velocity vector $\left[\mathrm{m} \cdot \mathrm{s}^{-1}\right]$

$\mathbf{u}_{b}^{\prime} \quad$ velocity vector of bubble turbulence $\left[\mathrm{m} \cdot \mathrm{s}^{-1}\right]$

$\mathbf{u}_{L}^{\prime} \quad$ vector of turbulence independent of bubble turbulence $\quad\left[\mathrm{m} \cdot \mathrm{s}^{-1}\right]$

$U_{G}:$ superficial gas velocity $\left[\mathrm{m} \cdot \mathrm{s}^{-1}\right]$

$x: \quad$ Cartesian coordinate $[\mathrm{m}]$

$y:$ Cartesian coordinate $[\mathrm{m}]$

$z:$ axial coordinate $[\mathrm{m}]$

$\alpha: \quad$ parameter defined by $\alpha=\Lambda_{c} / \Lambda_{l}$

$\varepsilon: \quad$ gas hold up defined by $\varepsilon=\left(1-\Lambda_{l}\right) / \Lambda \quad[-]$

$\varepsilon_{0}: \quad$ gas holdup at column center $\quad[-]$

$\varepsilon_{W}:$ gas holdup at column wall $[-]$

$\varepsilon_{m}: \quad$ cross sectional average of gas holdup [-]

$\Lambda: \quad$ duration for time averaging $[s]$

$\Lambda_{b}:$ total time during which bulk phase is observed $[s]$

$\Lambda_{c}:$ total time during which cloud phase is observed $[s]$

$\Lambda_{l}:$ total time during which liquid phase is observed $[s]$

$\xi$ : gradient vector at surface $\left[s \cdot \mathrm{m}^{-1}\right]$

$\boldsymbol{\tau}: \quad$ tensor of shear stress $\quad\left[\mathrm{Pa} \cdot \mathrm{m}^{-1}\right]$

$\phi$ : dimensionless radial coordinate defined by $\phi=r / R \quad[-]$

\section{Suffixes and other notations}

$b: \quad$ bulk phase or bubble turbulence

$c$ : $\quad$ cloud phase

$l: \quad$ liquid phase

$T_{i}^{a}: \quad$ value at surface $t=T_{i}^{a}$

$T_{i}^{l}: \quad$ value at surface $t=T_{i}^{l}$

$T_{j}^{\prime a}: \quad$ value at surface $t=T_{j}^{\prime a}$ 
$T_{j}^{\prime l}: \quad$ value at surface $t=T_{j}^{\prime l}$

${ }^{-b}$ : time-averaged value for duration $\Lambda_{b}$

${ }^{-c}$ : time-averaged value for duration $\Lambda_{c}$

${ }^{-l}: \quad$ time-averaged value for duration $\Lambda_{l}$

$-\Lambda$ : time-averaged value for duration $\Lambda$

$\int d t$ : sum of the time integration for individual time integral during which the $\Lambda_{c}$

cloud phase is continuously observed

\section{References}

[1] Degaleesan, S., Fluid dynamic measurements and modelling of liquid mixing in bubble column, PhD Thesis, Washington University, St. Louis, USA, 1997

[2] Hills, J.H., Radial non-uniformity of velocity and voidage in a bubble column, Trans. Inst. Chem. Engrs., 52, 1-10, 1974.

[3] Katoh, Y., M. Nishinaka and S. Morooka, Gas holdup distribution in bubble columns, Kagakukogaku Ronbunshu, 1,530-535,1975.

[4] Kurose, R., R. Misumi and S. Komori, Drag and lift forces acting on a spherical bubble in a linear shear flow, Int. J. Multiphase Flow, 27, 12471258, 2001.

[5] Legendre, D. and J. Magnaudet, The lft force on a spherical bubble in a viscous linear flow, J. Fluid Mech., 368, 81-126, 1998.

[6] Sato, Y., M. Sadatomi and K. Sekoguchi, Momentum and heat transfer in two-phase bubble flow, Int. J. Multiphase Flow, 7, 167-177, 1981.

[7] Tabib, M. V., S. A. Roy and J. B. Joshi, CFD simulation of bubble column -an analysis of interphase force and turbulence model, Chem. Eng. Journal, 139, 589-614(2008).

[8] Tomiyama, A., Y. Nakahara, Y. Adachi and S. Hosokawa, Shapes and rising velocity of single bubbles rising through an inner subchannel, $J$. Nuclear Science and Technology, 40, 136-142, 2003

[9] Ueyama, K. and T. Miyauchi, Time-averaged Navier-Stokes equations as Basic Equations for multiphase flow, Kagakukogaku Ronbunshu, 2, 595601, 1976.

[10] Ueyama, K. and T. Miyauchi, Behavior of bubbles and liquid in a bubble column, Kagakukogaku Ronbunshu, 3, 19-23, 1977.

[11] Ueyama, K., Study on gas-liquid multiphase flow in bubble columns, Doctoral thesis, University of Tokyo, Tokyo, Japan, 1978.

[12] Ueyama, K. and T. Miyauchi, Properties of recirculating turbulent two phase flow in gas bubble columns, AIChEJ., 25, 258-266, 1979. 
[13] Ueyama, K., Force balance controlling radial gas holdup distribution for the recirculating turbulent flow in bubble columns, J. Chem. Eng. Japan, 39, 16, 2006.

[14] Ueyama, K and M. Saitoh, Overview of multiphase flow phenomena in moving time-averaged space, Chem. Eng. Technol., 29, 1102-1106, 2006.

[15] Yamagoshi, T., Bachelor Thesis, Study on bubble columns, University of Tokyo, Tokyo, Japan, 1969.

[16] Zhang, X. and G. Ahmadi, Eulerian-Lagrangian simulations of liquid-gassolid flows in three-phase slurry reactors, Chem. Eng. Sci., 60.5089-5104, 2005 . 